\title{
Editorial
}

\section{Preface: Workshop on Advances in Optimization}

This issue contains a selection of research articles, most of them presented at the conference entitled Workshop on Advances in Optimization that was held in honor of Professor Shinji Mizuno on the occasion of his 60th birthday. The conference took place from August 12-13, 2016 at TKP Shinagawa Conference Center, Tokyo, Japan.

The research articles in this issue reflect the outstanding contributions that Shinji Mizuno has made to optimization as well as to related fields such as engineering, mathematics, and economics. The reader will find recent results in global optimization, linear optimization, semidefinite optimization, approximation algorithms, data mining and graphs.

We are grateful to the authors for their high quality contributions and to the referees for their thorough reviews. We also thank Editor-in-Chief Endre Boros and Publication Managers Katie D'Agosta and Ann Pulido for their kind help throughout the process of assembling this issue.

Shinji Mizuno was born in 1956 in Aichi, Japan. After finishing his B.S. in 1979, he received his Ph.D. in 1984 under the supervision of Masakazu Kojima at the Tokyo Institute of Technology. He started his academic career as assistant professor at the Chiba Institute of Technology (1984-1986) and the Tokyo Institute of Technology (1986-1990), and joined the Institute of Statistical Mathematics (1990-1999) as associate professor. He then returned to the Tokyo Institute of Technology in 1999 where he has been a full professor since 2001.

While Shinji's initial research interests were polynomial systems and homotopy equations, in the early 1990s he started his work on the interior point methods for linear programming problems and their generalization to linear complementarity problems. Many of his contributions are highly cited and have a long lasting impact that has been recognized by a number of awards. Jointly with Masakazu Kojima, Toshihito Noma, Nimrod Megiddo, and Akiko Yoshise, he was awarded the Lanchester Prize from the Operations Research Society of America in 1992 for a long-term research program aimed at establishing a theoretical foundation for primal-dual interior point methods for linear programming and linear complementarity problems. At that time, he was a recipient of the Alexander von Humboldt Foundation fellowship at Würzburg University, Germany. In addition, he received the Best Research Paper Prize from the Operations Research Society of Japan in 1991, and the 11th Japan IBM Scientific Prize in 1997.

Shinji's recent research interests include the simplex method, another fundamental method for solving linear programming problems, in collaboration with Tomonari Kitahara, and approximation algorithms for combinatorial optimization problems in collaboration with Yotaro Takazawa. He is interested in high-performance algorithms and optimization modelings for solving real-world problems such as container terminal operation and annual pension system in collaboration with practitioners.

The impact of his work is also reflected by the nearly 800 citations of his publications and a dozen achieving over 100 citations. He authored the highly regarded books Interior Point Methods of Mathematical Programming, Kluwer (1996) and Interior Point Methods, Asakura Shoten (2001). Shinji has been the chair of the 2016 International Conference on Continuous Optimization in Tokyo; a fellow of the Operation Research Society of Japan; and a plenary speaker for many international conferences including ICOTA: International Conference on Optimization: Techniques and Applications (2010), ICOTA (2013), POC: Pacific Optimization Conference (2104), COIA: International Conference on Control and Optimization with Industrial Applications (2015), and POC (2017).

Shinji's work and career were influenced by great colleagues and friends such as Florian Jarre, Masakazu Kojima, Nimrod Megiddo, Michael Todd, Yinyu Ye, and Akiko Yoshise. His supervisees are highly successful in both academia and industry.

We are delighted to dedicate this issue to a dear friend and mentor in recognition of his highly successful career and his important contributions to optimization.

Happy Birthday Shinji! 


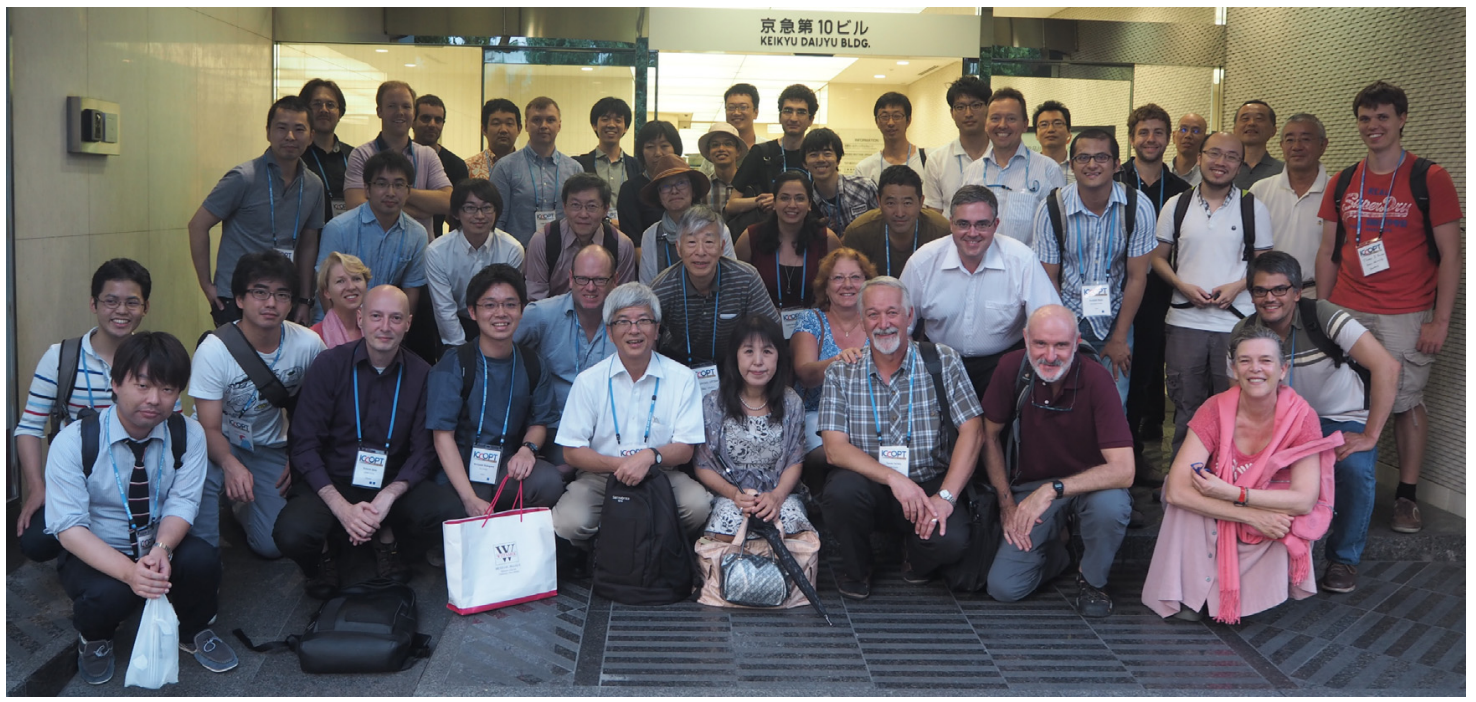

Backward: Tomohiko Mizutani, François Glineur, Yoshio Sano, Michael Metel, George Manoussakis, Yoichi Izunaga, Yoshio Okamoto, Sergei Chubanov, Kim Chuan Toh, Kei Takemura, Sunyoung Kim, Akiko Yoshise, Makoto Yamashita, Shunsuke Hayashi, Bruno Lourenço, Fatma Kılınç-Karzan, Shogo Kishimoto, Mitsuhiro Fukuda, Masakazu Muramatsu, Keiichi Morikuni, Etienne de Klerk, Kazuhide Nakata, Amitabh Basu, Aaron Sidford, Jianming Shi, Yin Tat Lee, Akihisa Tamura, Naohiko Arima, Thomas Dueholm Hansen.

Forward: Ken Kobayashi, Yuichi Takano, Mirai Tanaka, Gili Deza, Antoine Deza, Noriyoshi Sukegawa, David Bremner, Masakazu Kojima, Shinji Mizuno, Mioko Mizuno, Gabriella Terlaky, Tamás Terlaky, Miguel Anjos, Shmuel Onn, Ruth Onn, Julio Góez. (Picture taken by Tomonari Kitahara.)

Antoine Deza* Advanced Optimization Laboratory, Faculty of Engineering, McMaster University, Hamilton, Ontario, Canada E-mail address: deza@mcmaster.ca.

Tomonari Kitahara Faculty of Economics, Kyushu University, Fukuoka, Japan E-mail address: tomonari.kitahara@econ.kyushu-u.ac.jp.

Noriyoshi Sukegawa Department of Information and Computer Technology, Faculty of Engineering, Tokyo University of Science, Tokyo, Japan E-mail address: sukegawa@rs.tus.ac.jp. 